\title{
Ultra Long Integer Multiplication on GDPS
}

\author{
Y K Viswanadham \\ Dept of CSE \\ $S$ V University \\ Tirupati - 517 502, India
}

\author{
Dr Ch D V Subba Rao \\ Dept of Computer Sci Engg \\ $S$ V University \\ Tirupati - 517 502, India
}

\author{
T V Subrahmanyam \\ Dept of IT, Gudlavalleru \\ Engineering College, \\ Gudlavalleru-521 356, India
}

\begin{abstract}
Many Internet applications require intensive cryptographic calculation such as public-key encryptions and digital signatures. These schemes require a computation of large integer multiplications. Those cryptographic schemes are vulnerable to a brute-force attack, and the large key is the countermeasure. In practice, the key size that makes brute-force attack impractical will slows down the speed of encryption and decryption. Multiplication of two very long integers usually takes time to compute. Distributed Karatsuba algorithm is proposed to reduce the time of multiplication of two very long digits. The proposed architecture that makes use of Karatsuba algorithm achieves faster multiplication.
\end{abstract}

\section{Keywords}

GDPS, IMDP, Karatsuba Algorithm.

\section{INTRODUCTION}

Distributed computing is the process of aggregating the power of several computers to collaboratively run a single computational task in a transparent and coherent way. In other words it enables usage of the idle time of large numbers of networked computers to work on projects too large for any single group[7]. Recent distributed computing projects have been designed to use the computers of hundreds of thousands of volunteers all over the world, via the Internet, to look for extra-terrestrial radio signals [8], investigate and reduce uncertainties in climate modeling [9], detect earthquakes [10], prime numbers so large that they have more than ten million digits [11] etc. These projects require so much computing power to solve and they would be impossible for any single computer to solve in a reasonable amount of time.

\subsection{Cryptography}

During this time when the Internet provides essential communication between tens of millions of people and is being increasingly used as a tool for commerce, security becomes a tremendously important issue to deal with. There are many aspects to security and many applications, ranging from secure commerce and payments to private communications and protecting passwords. One essential aspect for secure communications is that of cryptography. But it is important to note that while cryptography is necessary for secure communications, it is not by itself sufficient. This field required very fast large integer multiplication. Multiplication of large integers is a fundamental requirement in polynomial multiplication for signal processing, coding theory [1].

\section{PROBLEM DEFINITION}

Multiplication is one of the most frequently used arithmetic operations in public key cryptography and the performance of a cryptosystem often depends mostly on the efficiency of a multiplication operation. The efficiency of cryptosystem is often determined based on how fast an encryption/decryption operation can be done. Modular exponentiation is hard operation used in most cryptosystems. It consists of performing modular multiplication repeatedly. Modular multiplication can be performed by first multiplying then reducing or by interleaving the multiplication and reduction steps. The former way is preferred as there are very fast multiplication algorithms as they were over studied while the latter is used when there is only a limited storage in the case of smartcards.

Multiplication of long integers is cornerstone primitive in most public-key cryptosystems. These kinds of multiplications are also required in mathematics, signal processing and astrophysics [1]. Multiplication of large numbers requires huge resource of time and space. The study of fast multiplication algorithms is always an important task pursued by mathematicians and computer scientists.

\subsection{Existing Systems}

The multiplication of ultra long integer can not be handled by a single computer in reasonable amount of time, but such kind of problems can be solved by Supercomputer.

\subsection{Limitations of Existing System}

The super computers may be fast, however those are not widely available because of its huge cost and maintenance. The simple client-server system may not achieve the speed of the super computer.

\section{PROPOSED SOLUTION}

The main objective of this proposal is multiplying the two ultralarge numbers (12000 digits) using low cost computing resources and getting faster, accurate result. Generalized Distributed Processing System (GDPS), which aims at providing a simple, generic, reusable and extensible platform for the purpose of Internet, based Massively Distributed Processing (IMDP). These systems are used to solve scientific problems which are too large to be solved by even the most powerful Supercomputers. In the Internet world there are so many computers having idle processing. So we can use such kind of idle CPU processing time by activate our own program on the idle client. For example 
SETI@Home[8], Predictor @ Home [6]...etc, provide for solving huge data analysis problems in a Distributed Environment.

\subsection{Methodology}

We propose GDPS for solving ultra long integer multiplication using Karatsuba algorithm. With this we can achieve faster result. It is an economical solution, because large volumes of computing resources are available on the internet. We can form those resources as a community and use them in their idle time as background process of our screensaver. Any Solution to Distributed Processing must provide the following:

- The Breakdown of Large tasks or Jobs into smaller independently possible portions or subtasks.

- The Allocation of these subtasks to the Participant Computers.

- The Physical Distribution of these tasks to the Participant Systems.

- $\quad$ The Processing of these subtasks by the relevant systems.

- The Physical Collection of partial results from the Participant Computers.

- The Combination of the partial solutions thus received into a Complete Solution.

\subsubsection{Server Side}

The server side architecture is as illustrated in the Figure 1.

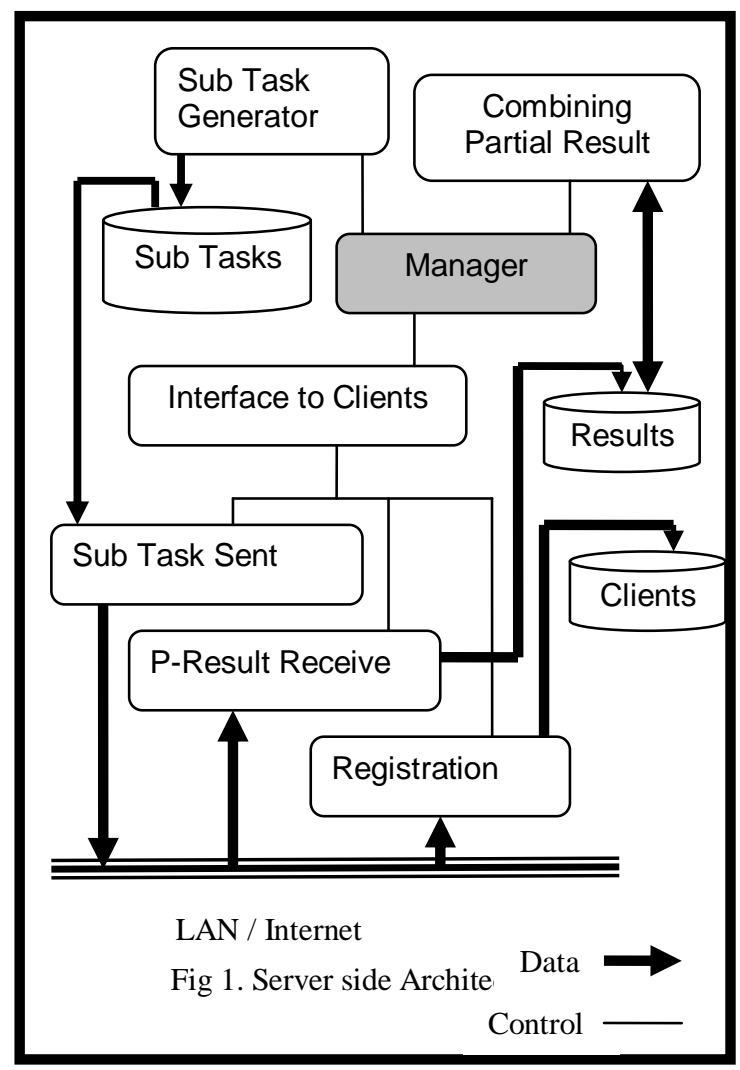

Fig 1. Server side Architecture

\section{Manager}

This is the Most Important Component of the Server End Architecture. It accesses the Sub Task, Partial Result and Client
Databases, and interfaces with the Internet Interface, The Sub task Generator and the Combining partial result Algorithms.

\section{The partial result combination algorithm interface}

This involves combining the partial result into final result and it is imitated by manager after receiving all partial results.

\section{The subtask generator algorithm interface}

This involves provision of a program call to the end user whereby he may create and store a sub task in the subtask database.

\section{The internet interface}

The Internet Interface has modules to handle the server end databases. The Server end of GDPS bears 3 databases which may be implemented using any standard database package or as files. They are

- A Database of the Various Subtasks or Work Units yet to be assigned to volunteers. This is a file handled by the subtask generator and updated or flagged when work units are assigned.

- A Database of the partial result for each subtask. All received result fragments are added here. Once all fragments for a subtask are received, the combination algorithm is initiated and these results are passed to it.

- A Database of clients who involved in this evaluation.

\subsubsection{Client Side}

The client side architecture is illustrated in the figure 2

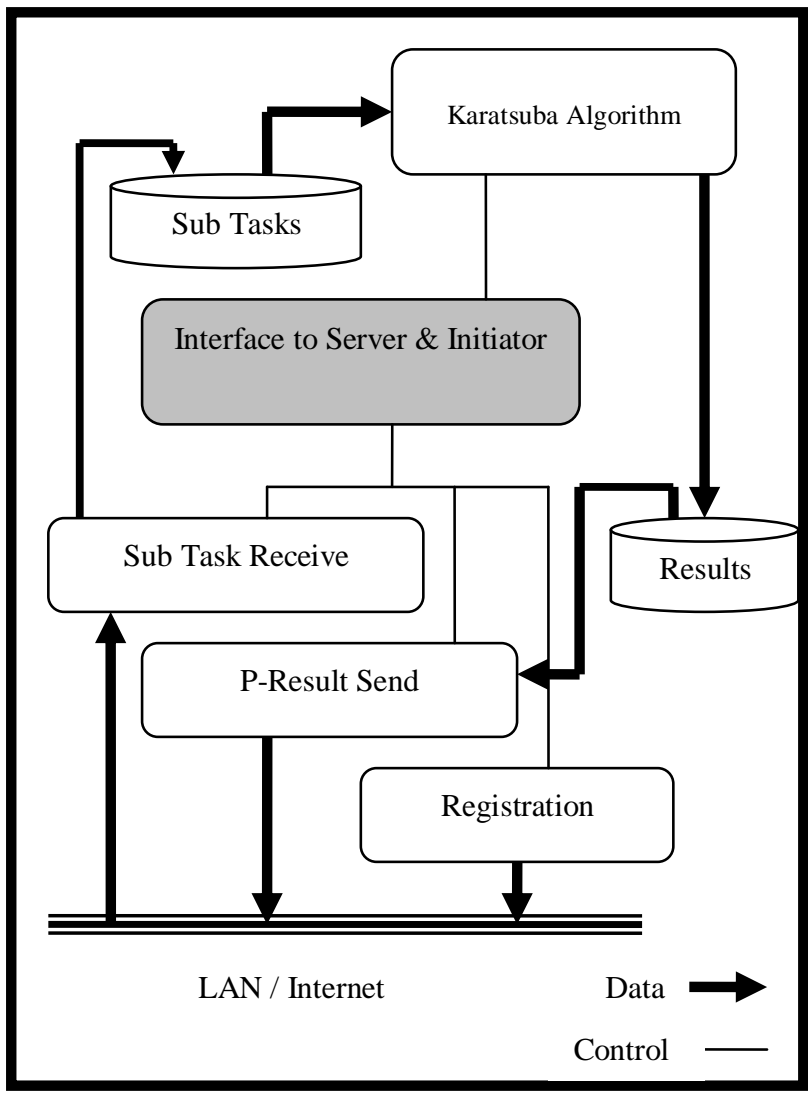

Fig 2. Client side Architecture

The Client End Algorithm Initiator

This component detects the activation of the Screensaver in Windows based PCs or waits for an explicit start up on other 
systems. It then initiates the relevant client end algorithms for subtask on the client PC.

\section{The Internet Interface}

This detects the initiation of an Internet Connection and proceeds to upload partial result and download more subtasks. The Client end PCs may not have standard database systems available and hence all data would be stored in files on the Clients.

\subsection{Karatsuba Algorithm}

In 1962 Karatsuba developed an algorithm for multiplying two numbers by using a divide-and-conquer method [2].Suppose two $n$-digit radix $b$ numbers $X$ and $Y$ are expressed as $x_{1} b^{n / 2}+x_{0}$ and $\mathrm{y}_{1} \mathrm{~b}^{\mathrm{n} / 2}+\mathrm{y}_{0}$ respectively.

The grade-school method computes the product $\mathrm{Z}$ of $\mathrm{X}$ and $\mathrm{Y}$ as follows:

$$
\begin{aligned}
Z & =X . Y \\
& =\left(x_{1} b^{n / 2}+x_{0}\right)\left(y_{1} b^{n / 2}+y_{0}\right) \\
& =x_{1} y_{1} b^{n}+\left(x_{1} y_{0}+x_{0} y_{1}\right) b^{n / 2}+x_{0} y_{0}
\end{aligned}
$$

Karatsuba proposed a multiplication algorithm that computes the product $\mathrm{Z}$ of $\mathrm{X}$ and $\mathrm{Y}$ as follows:

$$
\begin{aligned}
Z= & X \cdot Y \\
= & \left(x_{1} \dot{b}^{n / 2}+x_{0}\right)\left(y_{1} b^{n / 2}+y_{0}\right) \\
= & x_{1} y_{1} b^{n} \\
& +\left[\left(x_{1}+x_{0}\right)\left(y_{1}+y_{0}\right)-x_{1} y_{1}-x_{0} y_{0}\right] b^{n / 2} \\
& +x_{0} y_{0}
\end{aligned}
$$

To multiply two $\mathrm{n}$ digit numbers, the grade-school method has four $\mathrm{n} / 2$ digit multiplications and Karatsuba Algorithm has three $\mathrm{n} / 2$ digit multiplications [3].

$$
\begin{aligned}
\mathrm{T}(\mathrm{n}) & =4 \mathrm{~T}(\mathrm{n} / 2)+\mathrm{cn} \\
& =\mathrm{n}^{\log 4}{ }^{2} \\
& =\mathrm{n}^{2}
\end{aligned}
$$

For Karatsuba Algorithm

$$
\begin{aligned}
\mathrm{T}(1) & =1 \\
\mathrm{~T}(\mathrm{n}) & =3 \mathrm{~T}(\mathrm{n} / 2)+\mathrm{cn} \\
& =\mathrm{n} \log _{2}^{3} \\
& =\mathrm{n}^{1.58}
\end{aligned}
$$

\section{EXPERIMENTAL RESULTS}

The resulting program is implemented on Intel Core2Duo 2.0 $\mathrm{GHz}$ Processor with Jdk1.6.0 programming language. A graphical user interface is designed with swings to facilitate the user give the input in file mode as well as manually. The given input is divided into no. of segments which is a fixed length of digits. Those segments will be given as subtasks to clients. Those clients will send the result back to the server and stored in file. Finally combining algorithm will conquer the partial result and gives the final result.

It is tested for various combinations of size and no. of clients. The experimental results are analyzed in Fig- 3 \& 4 and noticed that best performance is only possible with no. of clients with respect to size of the problem. The increase of no. of clients for small problem degrades the performance of the system due to overhead. So the no. of clients should directly proportional to the Length of the problem up to threshold value.

\subsection{Performance Analysis}

The experiments have conducted on LAN in idle condition; all clients have the same specification Intel Core2Duo 2.0 GHz Processor 1 GB RAM.

\title{
Table 1: Experimental Results(Time ms)
}

\author{
For school book method
}

$\mathrm{T}(1)=1$

\begin{tabular}{|c|c|c|c|c|c|c|r|}
\hline $\begin{array}{c}\text { No.of Clients } \\
\text { I Length }\end{array}$ & $\mathbf{1}$ & $\mathbf{2}$ & $\mathbf{3}$ & $\mathbf{4}$ & $\mathbf{5}$ & $\mathbf{6}$ & $\mathbf{7}$ \\
\hline $\mathbf{1 0 0}$ & 235 & 63 & 31 & 62 & 78 & 94 & 104 \\
\hline $\mathbf{2 0 0}$ & 203 & 141 & 140 & 78 & 79 & 102 & 124 \\
\hline $\mathbf{3 0 0}$ & 438 & 313 & 234 & 157 & 188 & 218 & 252 \\
\hline $\mathbf{4 0 0}$ & 656 & 594 & 531 & 391 & 203 & 297 & 341 \\
\hline $\mathbf{5 0 0}$ & 812 & 766 & 656 & 437 & 302 & 406 & 512 \\
\hline $\mathbf{6 0 0}$ & 1250 & 1047 & 1079 & 1046 & 1126 & 859 & 902 \\
\hline $\mathbf{7 0 0}$ & 1765 & 1594 & 1563 & 1078 & 1124 & 844 & 983 \\
\hline $\mathbf{8 0 0}$ & 1984 & 1687 & 1749 & 1797 & 1735 & 1579 & 1592 \\
\hline $\mathbf{9 0 0}$ & 3374 & 3250 & 3172 & 3156 & 3124 & 3117 & 3109 \\
\hline $\mathbf{1 0 0 0}$ & 5265 & 4937 & 4906 & 4829 & 4719 & 4680 & 4562 \\
\hline $\mathbf{1 5 0 0}$ & 10266 & 10204 & 10141 & 9859 & 9625 & 9214 & 8609 \\
\hline
\end{tabular}




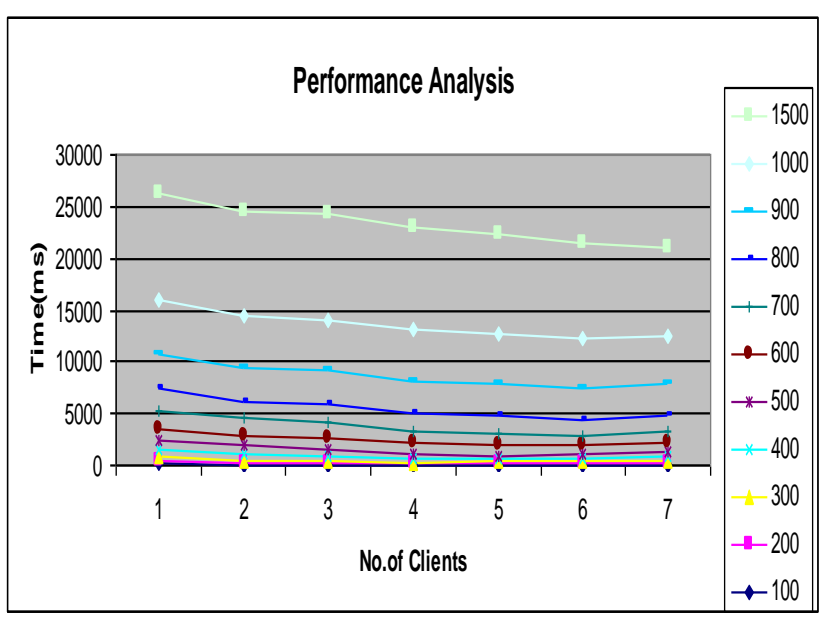

Fig 3.No.of Clients Vs Time for Size of the Problem

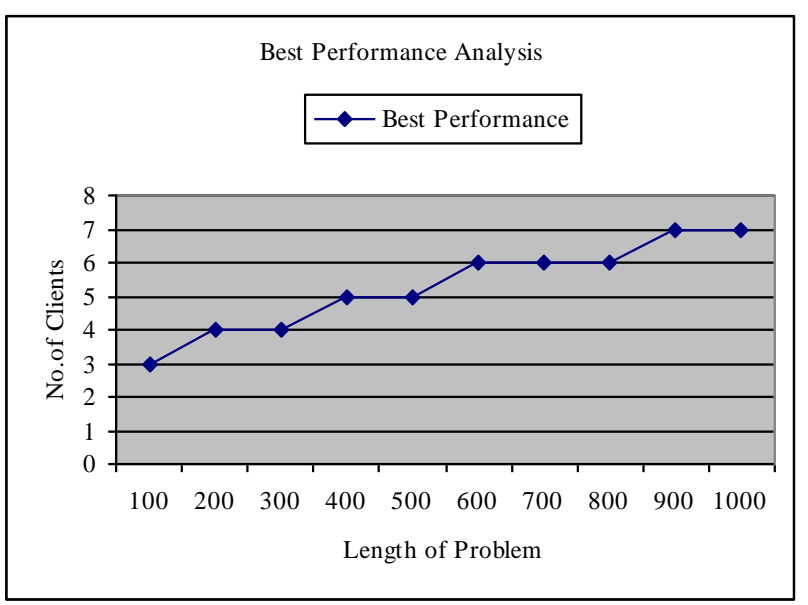

Fig.4: Length of the problem Vs No. of Clients

that will give us notable results. The fast multiplication approach can be used in prime number generation.

\section{REFERENCES}

[1] Andre Weimerskirch, "Generalizations of the Karatsuba Algorithm for Efficient Implementation”,http://www. Crypto.ruhr-uni-bochum.de/imperia/md/content/textekaweb.pdf, 2003

[2] A.Karatsuba and Y.Offman, "Multiplication of multi digit numbers on automata“, Soviet Physics-Doklady,1962.

[3] Chin-Bou Liu, "Design and Implementation of Long-Digit Karatsuba's Multiplication Algorithm Using Tensor Product Formulation", In The Ninth Workshop on Compiler Techniques for High-Performance Computing, 2003.

[4] Dan Zuras, "More On Squaring and Multiplying Large Integers", IEEE Transactions on Computers, AUGUST 1994

[5] J. Tudor, "Using the Parallel Karotsuba Algorithm For Long Integer Multiplication", European Conference on Parallel Processing,, 1997.

[6] M. Taufer, "A Protein Structure Prediction Supercomputer Based on Public-Resource Computing". IEEE Transactions on Parallel and Distributed Systems, Aug 2006.

[7] en.Wiktionary . org / wiki / distributed _ computing

[8] SETI@Home - setiathome.ssl.berkeley.edu

[9] Climate prediction - Climateprediction.net

[10 Quake-Catcher Network - qcn.stanford.edu

[11 math.html\#primegrid
6. FUTURE SCOPE

We are working on a new parallel implementation of the Karatsuba algorithm for handling division operation. We hope 